\title{
THE BOOK
}

\section{Reviewed by Daniel H. Ullman}

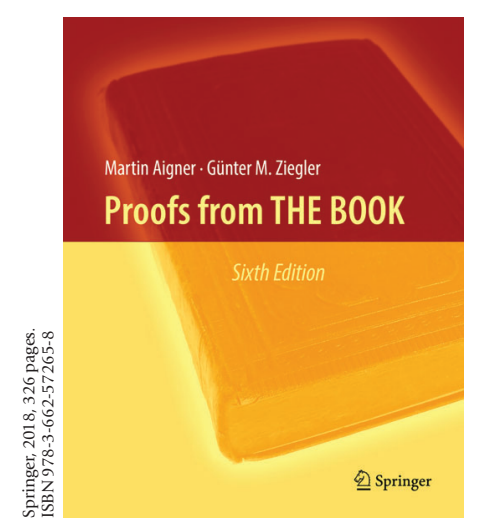

\author{
Proofs from THE BOOK \\ Sixth Edition \\ Martin Aigner and \\ Günter M. Ziegler
}

If you never did you should. These things are fun and fun is good.

—Theodore Seuss Geisel

Shortly after Paul Erdős died in 1997, Martin Aigner and Günter Ziegler published their book Proofs from THE BOOK (PFTB) dedicated to his memory. Now 20 years later, PFTB lives on in its sixth edition (2018), expanded and refined compared to the first edition, but unchanged in spirit. It is still published by Springer, still with the same cover design but for a change in color, and still in a nearly square rectangle of a binding designed conveniently to stick out farther than its neighbors on a bookshelf to facilitate extraction. (I wrote about the first edition of PFTB in the Notices in 1999 [1].)

The capitalized "BOOK" in the title is a reference to Erdős's idea that there is a collection of perfect proofs of the theorems of mathematics. But just as a proof is not a thing but an idea, so The Book is not a thing but an idea. One can conjure up its existence as a thought experiment, but we cannot know its contents. Perfection is not something human beings can produce or even identify. Aigner and Ziegler have collected in PFTB their best guesses as to what The Book might contain.

Daniel H. Ullman is a professor of mathematics at George Washington University. His email address is du11man@gwu. edu.

Communicated by Notices Book Review Editor Stephan Ramon Garcia.

For permission to reprint this article, please contact: reprint-permission aams.org.

DOI: https://dx.doi.org/10.1090/noti2322

\section{A Romantic View}

What does it even mean to say that a proof achieves perfection? In a certain view of mathematics, theorems are intended to be useful, and a proof is intended to afford a correct and infallible logical argument. In this utilitarian view, a proof is either correct or incorrect, and hence all correct proofs must be equally good. Where is there room for perfection on this binary scale?

It would betray Erdös's vision to argue that the purpose of a proof is merely to demonstrate the correctness of an assertion. Certainly mathematics can be useful, but it can also be beautiful. A perfect proof is more than a correct logical argument. There is also an aesthetic element. To experience a beautiful argument is to feel astonishment and joy, to become enlightened, to take pleasure in its ideas.

What exactly makes a proof beautiful? Beauty is in the eye of the beholder, so the cliché goes. But Immanuel Kant might have disagreed. He distinguished between beauty that is subject to the senses or the tastes of the beholder, like a delicious food or a work of art, and a beauty that is "disinterested," pleasing without appeal to desire. Kant understood how and why mathematicians use the word "beauty" to describe something that is absolute, permanent, and not subject to individual taste or even to the five senses.

It is not so easy to describe the essential elements of mathematical beauty. Trying to explain beauty is like trying to explain humor. But you know it when you see it. It involves an unexpected juxtaposition of ideas. It involves some element of surprise, like finding a key idea hiding in an unexpected place. The reader of a beautiful mathematical argument is suddenly enlightened, and experiences this beauty as an instantaneous heightening of knowledge and wisdom.

The beauty of a mathematical argument is, like mathematics itself, abstract. It is entirely unchanged when an argument is translated from one language to another. Moreover, it does not depend on elegance of language at all, as does, for example, the beauty of poetry. To see this, 
compare Euclid's famously beautiful proof of the infinitude of primes (the opening of the first chapter of PFTB) to the following parody, which I first heard from Michael Larsen several decades ago:

Claim. There are infinitely many composite numbers.

Proof. Suppose that $a_{1}, \ldots, a_{n}$ is a complete list of all composite numbers. It is easy to see that $n$ is at least 2. Let $M$ be the result of taking the product $a_{1} \cdots a_{n}$ and NOT adding 1 . The number $M$ is not prime, since it has $a_{n}$ as a nontrivial divisor. Also, $M$ is larger than $a_{n}$. Hence $M$ is a new composite number. This contradicts the fact that the list $a_{1}, \ldots, a_{n}$ is exhaustive.

This is a correct argument, and its rhetorical structure is analogous to the familiar proof of Euclid. Yet it is a ridiculous piece of mathematics, comical in its dimwittedness. By contrast, of Euclid's original proof, G. H. Hardy raved about its freshness and timelessness, writing famously in A Mathematician's Apology that "two thousand years have not written a wrinkle" on it.

Mathematics differs from other disciplines in one significant way. Should the universe come to an end, other disciplines will disappear along with it. Without the universe, there is no physics, no chemistry, no biology, no psychology, no sociology, no literature, no art, no music, no philosophy. And yet mathematics persists, entirely untouched by the apocalypse. The theorems remain true, and The Book remains intact, in any possible universe and one might say even in the absence of one. The robustness of mathematics comes from its essential abstraction. One need not have sheep to have positive integers. One need not have Rubik's cube to have group theory. One need not even have physical objects of any kind to have mathematics. Mathematics exists quite apart from the universe, and to call it "universal" is an understatement. The Book is not merely universal; it is permanent.

\section{The Book, The Book}

In PFTB, Aigner and Ziegler don't merely present beautiful arguments for the reader. They go out of their way to help the reader to see what is beautiful and why. This kind of editorializing is uncharacteristic in mathematical writing, but that is a shame. I wish this kind of writing were more customary. Readers of mathematics typically want to know not only what sentences comprise a proof but also the context for the result, how the proof was discovered, where the key steps lie, which shortcuts fail, and how to picture the idea. This kind of exposition is refreshing and appropriate and well illustrated by Aigner and Ziegler. They often explicitly identify key insights and surprising elements. It is a book created to help readers learn beautiful mathematics.

A look at some of the language in PFTB demonstrates why it is such a delight to read. Words like "gem," "jewel," "elegant," "surprising," "clever," and "striking" appear often. Love of mathematics shines through. From Chapter 6: "One proof stands out for its simplicity and elegance.
It was found by Ernst Witt in 1931 and combines two elementary ideas towards a glorious finish." Chapter 8: "we now courageously assume that" (a spectacular use of an adverb). Chapter 9: "After these two proofs ... we can't resist the temptation to present another, entirely different and completely elementary proof." From Chapter 29: "to prove it can turn out to be a tantalizing task-unless you open some magic door and everything becomes clear and simple." And from Chapter 41: "Now we are ready for the last proof, which may be the most beautiful of them all. Its origin is not clear; we got it from Stephan Brandt, who heard it in Oberwolfach. It may be 'folklore'..."

The wide margins of PFTB leave room for diagrams, background material, and a number of humorous cartoons by Karl Heinrich Hofmann.

PFTB has grown since its first edition, from 199 pages to 326, from 30 chapters to 45 . Most of the original edition remains in the present edition, although a chapter on the impossibility of arranging 13 unit balls all touching a 14th unit ball has been eliminated. In that chapter in the first edition, the authors write "It is the only proof in this book which involves a certain amount of explicit calculations with numbers." Perhaps it was deleted for just this reason. Or perhaps it is gone because the authors doubted whether the proof they exhibit is in fact the one from The Book.

Sixteen of the 45 chapters in the sixth edition are new since the first edition. They cover

- the law of quadratic reciprocity, which the authors claim is the result in the mathematical canon with the most proofs;

- Hadamard matrices (square matrices with entries in $\{1,-1\}$ that maximize determinant);

- the $p$-series with $p=2$ (they give four proofs that the sum is $\left.\pi^{2} / 6\right)$;

- the Borromean rings (they prove that this link is not achievable with rigid circles);

- the fundamental theorem of algebra (ironically in the section of PFTB on analysis);

- dissecting squares into an odd number of triangles of equal area (it's impossible);

- Van der Waerden's permanent conjecture (now a theorem, which states that the largest permanent among $n$-by- $n$ doubly stochastic matrices is achieved by the matrix all of whose entries are $1 / n$ );

- a cotangent expansion (in which an amazing series expansion is proved by what is called the "Herglotz trick");

- the Buffon needle problem (no calculus required!);

- tiling rectangles by rectangles (in which it is proved that if every tile has an integer side, then the entire assembled rectangle has an integer side, patterned after a beautiful paper of Stan Wagon in the American Mathematical Monthly);

- shuffling cards (based on a paper of David Aldous and Persi Diaconis in the American Mathematical Monthly); 
- lattice paths (following work of Ira Gessel and Gerard Viennot);

- identities versus bijections (about integer partitions and pentagonal numbers);

- the Kakeya problem (in which a classical problem is extended from Euclidean space to finite fields and a proof from the 2008 doctoral dissertation of Zeev Dvir is explicated);

- permanents and entropy (in which an upper bound for the permanent is presented); and

- the chromatic number of the Kneser graphs (presenting a 2002 short proof by Joshua Green that appeared in the American Mathematical Monthly).

This list makes clear that, while PFTB explores a wide spectrum of mathematical areas, the authors have their favorites. The new edition is organized into five areas (number theory, geometry, analysis, combinatorics, and graph theory) just as the first edition was, but combinatorial results and arguments stand out. Naturally, the selection of chapters and arguments represents the expertise, experience, and biases of the authors. For example, several chapters involve determinants and permanents. Also, where the book delves into continuous mathematics, the results are generally well known (e.g., the fundamental theorem of algebra, the irrationality of $e$ ), while the results in discrete mathematics are often less well known (e.g., the Kakeya problem on finite fields, completing partial Latin squares).

\section{A Practical View}

In the end, the view that mathematical beauty is inherent and immutable is a romantic vision, but it probably does not hold up to scrutiny. How could a chapter on 13 unit balls be beautiful in 1998 but no longer so in 2018 ? The answer is that beauty is after all in the mind of the beholder. I surmise that the beauty of this result faded over two decades in the minds of the authors. A mathematical novice sees nothing beautiful at all in PFTB; it is all out of reach. Moreover, a mathematical expert may see nothing beautiful in a familiar, elementary argument that no longer contains any element of mystery or surprise. The beauty of a mathematical argument is invisible if you know too little or too much. It is a familiar experience for a mathematical student to be enthralled with a newly learned technique or argument, only later with more experience to regard the same technique or argument as routine or even mundane.

Here is an example of what I would call a beautiful proof. I first heard it from Ivan Niven, at an invited address at JMM some years ago. It does not appear in PFTB.

Theorem. The square root of 2 is irrational.

Proof. Were it not so, there would be a smallest positive integer $n$ such that $n \sqrt{2}$ is itself an integer. But that's impossible, since if $n$ were such an integer, then $n \sqrt{2}-n$ would be a smaller one.

While I find this argument unusually beautiful, it is hard for me to argue that it exhibits the disinterested beauty of
Kant. What makes it lovely in my mind is the contrast to the very well-known argument of Euclid that takes a few extra sentences to deliver. If you don't know that proof, then, well, never mind this one.

Another reason to love this proof is that the final punch line comes as a surprise, and at first you don't see it. But then comes the moment-wait for it! — when you suddenly see the point. When Niven delivered this proof in his talk, he paused, and the thousand or more mathematicians in attendance were utterly silent for about five seconds, after which they suddenly and simultaneously erupted into sustained laughter and applause. If you don't understand what needs to be checked and why, then, well, never mind again. And at the other end of the spectrum, if you've seen this argument before, the surprise is ruined.

Whether a piece of mathematics is beautiful depends on what mathematics you know. It may therefore be necessary to imagine that all mathematicians have their own Book, comprised of those arguments that delight them in the context of their mathematical background. I would hope that all mathematicians have experienced beauty in their work, but each mathematician will find that beauty in a different place.

Still, PFTB works very well on me. While Aigner and Ziegler reveal their biases in deciding what to include in PFTB, I have to admit that I generally share those biases. In these pages are so many gems and jewels, presented at the right level for me to enjoy. Any professional mathematician or any advanced undergraduate mathematics student will find much here to bask in. This is "mathematics for fun." If I am ever exiled to a desert island with one book to take along, PFTB will be my choice. Complete mastery of its pages would require many months, but the effort would be joyous and the result of that mastery would be profound wisdom.

\section{References}

[1] Daniel H. Ullman, Review of "Proofs from THE BOOK," Notices Amer. Math. Soc. 46 (1999), no. 7, 789-791.

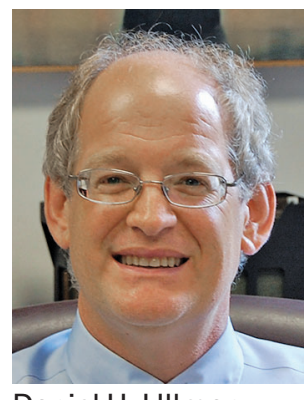

Daniel H. Ullman

\section{Credits}

Book cover image is courtesy of Springer Nature. Author photo is courtesy of the author. 